\title{
Wissenschaft braucht Zeit
}

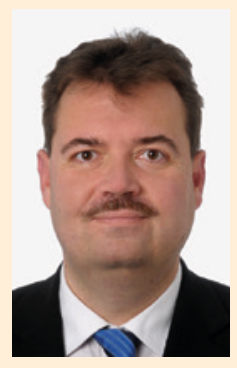

Liebe Kolleginnen und Kollegen,

2015 ist ein Jahr der Meilensteine in der Erforschung des Weltraums. Kürzlich gab die NASA die Entdeckung des Exoplaneten Kepler-452b bekannt, einem erdähnlichen Planeten in der habitablen Zone eines unserer Sonne ähnlichen Sternes. Seit Juni sendet der Lander Philae wieder vom Kometen Tschurjumow-Gerassimenko, wo er im November letzten Jahres nach einer mehr als 10-jährigen Reise zielgenau angekommen ist. Und im Juli erreichte die Raumsonde New Horizons nach 9 Jahren den Pluto. Bei der Erforschung des Weltalls werden Jahrzehnte als akzeptable Zeitgröße wahrgenommen, bei der terrestrischen Forschung sieht dies leider anders aus. Der Zeitbegriff in der Wissenschaft hat sich verändert.

Forschungsvorhaben über 10 oder gar 20 Jahre sind politisch kaum umsetzbar. Zeit für scheinbar Unmögliches oder für Irrtümer und Fehlschläge gilt als unproduktiv. Grundlagenforschung wird in der heutigen Zeit nicht mehr sui generis als nützlich akzeptiert. Wissenschaftler müssen sich immer mehr gegenüber Politik, Presse und Forschungsmanagern rechtfertigen und werden zu unsinnigen KostenNutzen-Abwägungen gezwungen, in denen der Faktor Zeit zu einer ökonomischen Messgröße wird. Heute arbeitet der Forscher unter den Zwang der Zeit. Erfolge müssen schnell und vor allem vorhersagbar sein. Risikoreiche oder gar „spekulative“ Vorhaben werden erst gar nicht erwogen. „Spekulativ“ ist zu einem Schimpfwort in der Wissenschaft geworden. Nur das Naheliegende, Logische und Planbare scheint seriös, die Suche nach dem Unbekannten oder „Ausprobieren“ gelten als unwissenschaftlich. Heute scheint man Forschern nicht mehr zu trauen, denn man kontrolliert sie durch detaillierte Forschungspläne, pseudopräzises Projektmanagement und eine Berichts- und Evaluationsbürokratie. Anscheinend scheint man es Forschern heute nicht mehr zuzutrauen, ohne „Management“ von außen erfolgreich zu sein.

Management setzt Ziele, Planbarkeit und Erfolgskontrollen voraus. Und hier wird es schwierig: Forschung, die nicht in kurzer Zeit zum „Erfolg“ führt, wird möglichst vermieden. „Erfolg“ wird heute mittels den bei Forschungsmanagern genauso beliebten wie untauglichen „impact factors“ quantifiziert. Leider sind nicht nur Forschungsmanager, sondern auch Wissenschaftlerkollegen schnell darin geworden, über Erfolg oder Wichtigkeit eines Forschungsresultats oder -themas zu urteilen. Kaum einer scheint mehr akzeptieren zu wollen, dass über die „Bedeutung“ von Forschung allein in der Zukunft entschieden wird. Prognosen sind schwierig, vor allem wenn sie die Zukunft betreffen, hatte Niels Bohr auf die Frage der Zukunft der Quantenphysik gesagt. Heute mehr denn je sollte Bescheidenheit wieder eine Tugend der Wissenschaft sein. Bescheidenheit im Angesicht dessen, wie wenig wir eigentlich wissen und wozu dieses Wenige in Zukunft gut sein kann.

Die raumfahrtmedizinischen Artikel dieser Ausgabe berichten über neue Erkenntnisse und Fortschritte auf den Gebieten der Hämostase, des Immunsystems, des muskuloskeletalen Trainings und der Konservierung von Blutprodukten. Gleichzeitig zeigen sie, auf wie viele ganz grundlegende Fragen noch keine Antwort gefunden wurde. Fortschritt entsteht dort, wo es mehr Fragen als Antworten gibt. Die Forschung wird Antworten finden, aber nur wenn man ihr die dafür notwendige Zeit gibt. Das können wir von der Raumfahrt lernen.

Ihr

Prof. Dr. Dr. Oliver Ullrich 